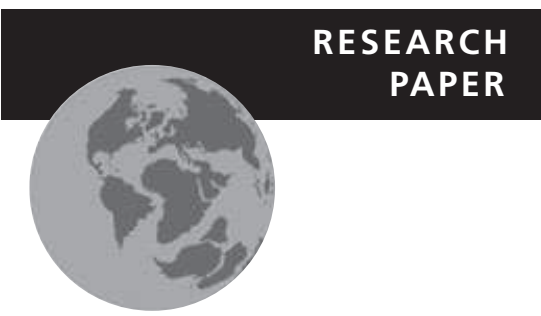

\title{
Mammal community structure correlates with arboreal heterogeneity in faunally and geographically diverse habitats: implications for community convergence
}

\author{
Julien Louys ${ }^{1 *}$, Carlo Meloro ${ }^{2}$, Sarah Elton ${ }^{2}$, Peter Ditchfield ${ }^{3}$ and
}

Laura C. Bishop ${ }^{1}$

${ }^{1}$ Research Centre in Evolutionary Anthropology and Palaeoecology, School of Natural Sciences and Psychology, Liverpool John Moores University, Byrom Street, Liverpool L3 3AF, UK, ${ }^{2}$ Functional Morphology and Evolution Unit, Hull York Medical School, University of Hull, Cottingham Road, Hull HU6 7RX, UK, ${ }^{3}$ Research Laboratory for Archaeology and the History of Art, School of Archaeology, University of Oxford, Dyson Perrins Building, South Parks Road, Oxford OX1 3QY, UK

${ }^{*}$ Correspondence: Julien Louys, School of Natural Sciences and Psychology, James Parsons Building, Liverpool John Moores University, Liverpool L3 3AF, UK.

E-mail: j.louys@ljmu.ac.uk

\section{ABSTRACT}

Aim To quantify how mammal community structure relates to heterogeneity of vegetation for palaeoecological reconstructions, and to test whether historical or environmental factors are more important in structuring communities.

Location Sixty-three natural protected areas in Asia, Africa and South and Central America.

Methods We defined faunal communities by allocating species to ecological guilds and calculating proportional representation within each guild. Vegetation heterogeneity for each natural protected area was calculated from satellite images. The relationship between these ecospaces was calculated using canonical correlations analysis, redundancy analysis and principal components analysis. We expected that large, herbivorous mammals would be most strongly correlated with open areas. Convergence was tested by independently eliminating the effects of geography and vegetation heterogeneity on the structure of the mammal communities. We expected that vegetation would more strongly structure communities than geographical position.

Results We show that the guild structure of communities across habitats is significantly correlated with vegetation heterogeneity. The highest correlation was between small, scansorial-arboreal secondary consumers and heavy tree cover. The first convergence analysis shows American communities distinguished from Asian and African communities; these latter communities show a remarkable convergence in structure. Historical factors only affected the continent whose mammals had experienced a long period of isolation. The second convergence analysis shows that almost all biomes have the same or very similar community structure regardless of continent.

Main conclusions Communities from the same environments in different continents showed remarkable convergence. Communities from the same continents only converged when those continents shared a recent geological and biological history. These results suggest that historical and environmental factors are operating over different timescales. This study confirms that environmental reconstructions made on the basis of whole communities will accurately reflect the environment that the community lived in. However, reconstructions made for fossil sites in deep time need to take historical factors into consideration. Small, arboreal and scansorial secondary consumers show the strongest correlation with vegetation, correlating with continuous tree canopy cover. This relationship allows simple reconstructions of the amount of tree cover occurring in a landscape from the proportion of species from the community falling in this ecological guild. 


\section{Keywords}

Africa, America, Asia, community convergence, guild, niche, palaeoecology, synecology.

\section{INTRODUCTION}

Palaeoecologists use two approaches to the fossil record to reconstruct past environments. The first approach (autecology) examines the palaeobiology of a single taxon through a variety of methods (e.g. isotopic analyses, ecomorphology) and uses the results to infer the environment inhabited by that species. The second approach (synecology) reconstructs environments on the basis of the relationship between communities and habitats. This method uses modern communities as analogues of fossil ones and thus relies on the correlation between environmental variables and community structure in the present (however, see Rodríguez et al., 2006, for a critique). This latter correlation has been widely reported and it applies to several animal groups, including mammals (e.g. Fleming, 1973; Danell et al., 1996; Olff et al., 2002). Abiotic factors such as precipitation, temperature and soil fertility have been shown to control the diversity of large mammalian herbivores, principally through their effects on plant abundance and quality (Fleming, 1973; Olff et al., 2002). Environmental factors such as climate, habitat and landscape heterogeneity are often cited as influencing the geographical gradients of species richness in both plants and animals (Kerr \& Packer, 1997; Francis \& Currie, 1998, 2003; Kerr et al., 2001; Currie et al., 2004). Two main aspects of vegetation structure that affect mammalian communities have been suggested: namely heterogeneity and vegetation complexity (August, 1983; Bakker \& Kelt, 2000; Williams et al., 2002). Heterogeneity refers to the lateral extent of vegetation coverage, while complexity refers to the vertical layering of vegetation (sensu August, 1983). Increased vegetation heterogeneity has been shown to correspond to increases in species diversity amongst small mammals (Williams \& Marsh, 1998). Vegetation heterogeneity is therefore likely to increase the representation of different kinds of mammalian species within an ecosystem (similarly, complexity would also increase representation; however, this is not dealt with further here).

Mammals occupy different ecological niches, and they can be grouped according to the resource they exploit as well as the way they do it (=guild sensu Root, 1967, and Blondel, 2003). A correlation between guild or niche diversity and/or proportionality and broadly described habitats has been reported by a number of researchers (Andrews et al., 1979, Nesbit-Evans et al., 1981; Andrews, 1996; Reed, 1998; Kovarovic et al., 2002; Mendoza et al., 2005; Louys \& Meijaard, 2010), and this relationship has been used extensively by palaeoecologists to reconstruct palaeohabitats (Andrews, 1989, 1996; Kay \& Madden, 1997; Reed, 1997; Mendoza et al., 2004, 2005; Reed \& Russak,
2009; Louys \& Meijaard, 2010). Although the nature of vegetation heterogeneity and complexity is implicit in many of these categorical classifications, they do not explicitly provide a quantitative measure of the structure of the vegetation in those habitats.

In addition to the influence of environmental factors, community structure and species richness gradients will also be a consequence of historical factors (Ricklefs, 1987, 2004, 2007; Blondel, 1991; Samuels \& Drake, 1997; Qian \& Ricklefs, 2004; Rodríguez et al., 2006). For example, human impacts, regional climatic and vegetational changes, the invasion of the North American biota, and differences in herbivore-vegetation dynamics have been cited as possible historical factors contributing to differences between South American and African faunal communities (Cristoffer \& Peres, 2003; de Vivo \& Carmignotto, 2004). Whether historical or environmental factors are more important in structuring communities has received much debate, and is often expressed as the theory of community convergence. This theory posits that although historical factors may control the initial species pool from which a community can be assembled, given enough evolutionary time all niches within a particular habitat will be filled (Fuentes, 1976; Andrews, 1996; see also Wilson et al., 1995; Wilson \& Whittaker, 1995). Therefore, communities drawn from two biogeographically distinct species pools, experiencing similar environmental conditions will, given sufficient evolutionary time, be more similar in structure to each other than to communities from the same biogeographical area inhabiting different environments (sensu Fuentes, 1976). The concept of community convergence is of great importance to palaeoecologists, as interpretation of past environments requires an understanding of the relationship between community structure and habitat (Andrews, 1996; Rodríguez, 2004, 2006; Rodríguez et al., 2006). However, the degree to which historical factors contribute to shaping communities is still not clear, and evidence for community convergence has been equivocal (Samuels \& Drake, 1997).

We examined the relationship between guild proportionality (i.e. the proportion of species from a given community falling in a defined guild, sensu Wilson, 1989) and arboreal heterogeneity (defined as the relative proportion of area falling under one of four discrete tree canopy categories; see Appendix S1 in Supporting Information) amongst faunally diverse, medium- and large-bodied mammalian communities, and explored the implications for community convergence. In order to do this, we compiled a database of ecological and vegetation variables for natural protected areas (NPAs) across three continents (Fig. 1, Table 1). Two ecospaces are described by separate variables for 


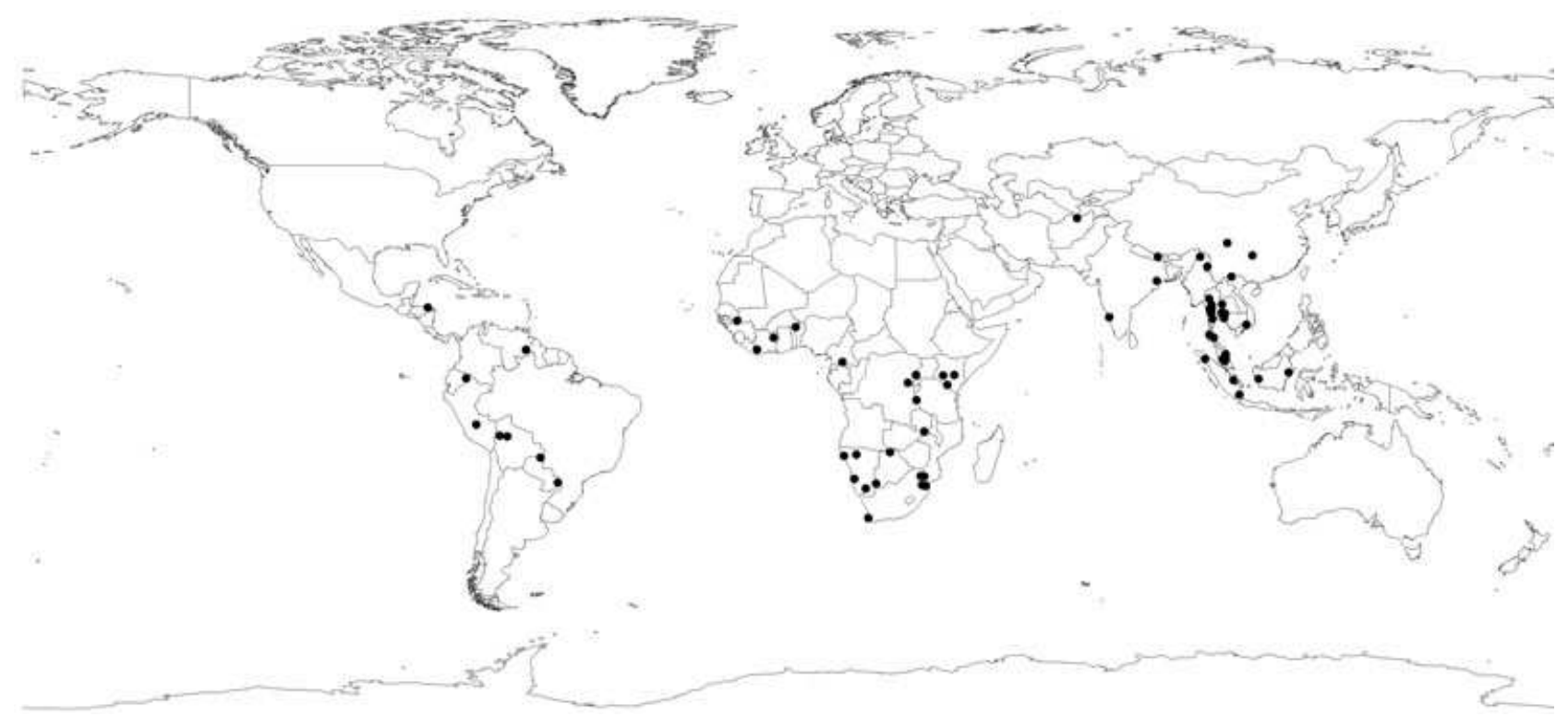

Figure 1 Geographical position of the natural protected areas examined in this study. For the list of natural protected area names, abbreviations and geographical coordinates see Appendix S1.

each protected area: the first by the ecological niche space occupied by mammals in the protected areas; the second by the relative proportions of discrete tree cover categories in the same areas, which we call here arboreal heterogeneity. These categories represent four discrete grades of canopy cover, from continuous to absent (see Appendix S1). We restricted our analyses to faunally diverse protected areas, standardising, to a degree, mammalian diversity in our samples. We further standardised the effects of species diversity by examining guild proportionality rather than number of species. We restricted our analyses to a fixed area regardless of NPA size, thereby minimising area effects.

We expect that arboreal heterogeneity will be strongly correlated with community structure. In particular, because of their dependence on trees, primary feeding, arboreal species should be more associated with dense canopy cover (Fleming et al., 1987). On the other hand, large terrestrial mammals as a whole are generally more indicative of open areas (e.g. Vrba, 1980). We also explored potential community convergence between the three continents examined. Two analyses were conducted. In the first analysis, we corrected for habitat (a proxy for environmental factors). This then allowed us to explore the influence of history on structuring communities. If mammal community structure differs between continents once environment has been accounted for, this would suggest that historical differences between continents are important in structuring communities. Conversely, if there is no separation between continents, this suggests other factors are involved in structuring communities. In the second analysis, we corrected community structure for geographical position (a proxy for history). This allowed us to explore the influence of environmental factors. If mammal community structure separates between broad environmental cat- egories (defined after Olson et al., 2001), this suggests that environmental differences are important in structuring communities. On the other hand, if there is no separation between habitats, this suggests that other factors are involved.

\section{MATERIALS AND METHODS}

All abbreviations used in this study are listed in Table 1. Species lists and geographical coordinates for each NPA from Africa $(n$ $=23)$, Asia $(n=32)$ and Central and South America (hereafter 'America') ( $n=8)$ were taken from the Man and the Biosphere Species Database (http://ice.ucdavis.edu/mab). The locations of these protected areas are shown in Fig. 1. Only species lists with more than 32 species were used; this number was calculated as the minimum number necessary to confidently distinguish between three discrete and broadly defined habitat types across ecosystems (namely closed, mixed and open) in multivariate synecological analyses (see Louys et al., 2009, for a detailed methodology and discussion of how this number was obtained).

\section{Ecological categories}

The two most often used functional gradients in mammalian palaeoecological studies are trophic and locomotor guilds (Andrews, 1996; Reed, 1998; Kovarovic et al., 2002; Rodríguez, 2004; Mendoza et al., 2005; Rodríguez et al., 2006). Within trophic guilds, primary and secondary consumers are generally subdivided into more distinct categories, such as hypercarnivore, insectivore or frugivore. Likewise for locomotor guilds, where arboreal or terrestrial categories can be subdivided into, for example, scansorial, fossorial and aquatic. These are also often broken down according to body weights to account 
Table 1 List of abbreviations.

\begin{tabular}{|c|c|c|c|}
\hline & \multicolumn{2}{|c|}{ Abbreviation } & \multirow{2}{*}{$\begin{array}{l}\text { Abbreviation } \\
\text { ONP }\end{array}$} \\
\hline Natural protected areas: & & Otishi National Park & \\
\hline Ajar Valley Wildlife Sanctuary & AJV & Pang Sida National Park & PSP \\
\hline Amboseli National Park & ANP & Parc National de la Comoe & PCO \\
\hline Berbak National Park & BNP & Parc National de Tai & PNT \\
\hline Bukit Kutu Wildlife Reserve & BKR & Parc National du Niokolo-Koba & PNK \\
\hline Cape West Coast Biosphere Reserve & CWC & Parque Nacional Canaima & PNC \\
\hline Cat Tien & CAT & Parque Nacional Iguazú & PNI \\
\hline Daweishan Nature Reserve & DNR & Pasoh Virgin Jungle Reserve & PVJ \\
\hline Erawan National Park & ERP & Phu Khieo Wildlife Sanctuary & PKS \\
\hline Estacion Biologica Beni & $\mathrm{EBB}$ & Queen Elizabeth (Rwenzori) National Park & QEP \\
\hline Etosha National Park & ENP & Reserva de la Biosfera de Yasuni & RBY \\
\hline Fanjingshan Mountain Biosphere Reserve & FMR & Reserve de la Biosphere de la Pendjari & RBP \\
\hline Gunung Leuser National Park & GLP & Reserve Forestiere et de Faune du Dja & RFD \\
\hline Gunung Palung National Park & GPP & Río Negro National Park & RNP \\
\hline Huai Kha Khaeng Wildlife Sanctuary & HKK & Río Plátano Biosphere Reserve & $\mathrm{RPB}$ \\
\hline Kaeng Krachan National Park & KKP & Royal Chitwan National Park & $\mathrm{RCP}$ \\
\hline Kahuzi-Biéga National Park & KBP & Simlipal Tiger Reserve & STR \\
\hline Kalahari Gemsbok National Park & KGP & Skeleton Coast Game Park & SCP \\
\hline Khao Luang National Park & KLN & South Luangwa National Park & SLP \\
\hline Khao Soi Dao Wildlife Sanctuary & KSD & Taman Negara National Park & TNP \\
\hline Khao Yai National Park & KYP & Thap Lan National Park & TLP \\
\hline Khlong Lan National Park & KLP & Thung Yai Naresuan Wildlife Sanctuary & TYN \\
\hline Khlong Saeng Wildlife Sanctuary & KSS & Way Kambas National Park & WKP \\
\hline Kora National Reserve & KNR & Wolong Nature Reserve & WNR \\
\hline Krau Wildlife Reserve & KWR & Ecological Guild & \\
\hline Kruger National Park & KNP & Small, arboreal primary consumer & BAP \\
\hline Kruger to Canyons Biosphere Reserve & KCR & Small, arboreal secondary consumer & BAS \\
\hline Kudremukh National Park & KUD & Small, terrestrial primary consumer & BTP \\
\hline Kutai National Park & KUP & Small, terrestrial secondary consumer & BTS \\
\hline Lake Nakuru National Park & LNP & Medium arboreal primary consumer & CAP \\
\hline Madidi National Park & MDP & Medium, arboreal secondary consumer & CAS \\
\hline Mahale Mountains National Park & MMP & Medium, terrestrial primary consumer & СТP \\
\hline Malolotja Nature Reserve & MAR & Medium, terrestrial secondary consumer & CTS \\
\hline Mamili Nature Reserve & MNR & Large, arboreal primary consumer & DAP \\
\hline Mlawula Nature Reserve & MLR & Large, arboreal secondary consumer & DAS \\
\hline Namdapha National Park & NAP & Large, terrestrial primary consumer & DTP \\
\hline Namib-Naukluft Park & NNP & Large, terrestrial secondary consumer & DTS \\
\hline Naute Dam & NAD & Very large, arboreal secondary consumer & EAS \\
\hline Nujiang Nature Reserve & NNR & Very large, terrestrial primary consumer & ETP \\
\hline Om Koi Wildlife Sanctuary & OKS & Very large, terrestrial secondary consumer & ETS \\
\hline
\end{tabular}

for feeding preferences and resource use (Andrews, 1996; Kay \& Madden, 1997; Reed, 1997, 1998; Mendoza et al., 2005). We restricted our categorization to three ecological dimensions, namely: (1) body mass - tiny (A, <1 kg), small (B, $1-10 \mathrm{~kg}$ ), medium (C, 10-45 kg), large (D, 45-180 kg) and very large (E, $>180 \mathrm{~kg}$ ); (2) trophic level - primary (P) or secondary (S) consumer; and (3) locomotion - terrestrial $(\mathrm{T})$ or potential and strict arboreality (A).

While these categorizations may seem very broad for extant taxa, they were chosen such that fossil taxa could be allocated to a guild with minimal errors (however, we note that even for extant taxa this level of ecological information was sometimes not available). Furthermore, the dichotomous definition of sub- strate use and diet allows for the classification of extinct species where only species lists may be available. This is particularly pertinent for fossil assemblages which have been lost, or where access to primary fossil material may be impossible due to political or financial reasons (Louys \& Meijaard, 2010). While the most accurate palaeosynecological analyses will always be made if an autecological reconstruction for each individual species is available, this situation is rarely the case. Autecological reconstructions of fossil species require detailed anatomical examination and/or chemical analyses, and well-preserved and nearcomplete fossil specimens. While analysing entire communities in this way should be lauded, resources will rarely be available for such an endeavour. Further, to compare between numerous 
extinct communities on the basis of more detailed ecological guilds requires that each species within each different community be subjected to such detailed analyses. These very broad categories therefore minimize misallocation to an ecological category while still allowing for correct retrodiction of habitat category (Louys \& Meijaard, 2010). They also facilitate large-scale comparisons between spatially and temporally disparate communities.

Ecological data for all species were taken from published records (Nowak, 1999), or where these were not available from the most closely related species within the same genus. Taxa with insufficient or ambiguous ecological information were excluded from the analysis $(n=6$; maximum $n$ for any NPA $=2$; see Appendix S1 for list). Assignment to body mass category was achieved on the basis of mean recorded body weights between males and females where listed; when only single body weights were provided, these were used. Where no weights were recorded, body size was estimated from body length. Because neoecological and palaeoecological information is unresolved for many micromammals, the tiny weight category $(\mathrm{A},<1 \mathrm{~kg}$ ) was excluded (see also Louys et al., 2009, and Louys \& Meijaard, 2010). We also follow other palaeoecologists in excluding bats from the synecological analyses (e.g. Andrews et al., 1979; Kay \& Madden, 1997; Rodríguez, 2004). Terrestrial species include all strictly terrestrial mammals together with partly aquatic species (e.g. Lutra lutra, the Eurasian otter), while 'arboreal' species included all others. Primary consumers were scored as those which had no dietary intake of animal foods. All others were scored as secondary consumers. Using the faunal lists for each protected area studied, each species was scored according to size, trophic and locomotor guild. These data were used to calculate the relative proportion of the 15 discrete ecological guilds for each protected area (see Appendix S1).

\section{Habitat classification}

Google Earth was used to capture a square area of $25 \mathrm{~km} \times$ $25 \mathrm{~km}$ centred on the geographical coordinates provided for each protected area. This area potentially, but not strictly, could be used by the animals on the protected area's faunal list. Each of the four different types of tree cover (heavy, moderate, light and no tree cover; Appendix S1), as well as cloud cover and water bodies were determined visually for each area, and a signature for each category was created for individual NPAs using ARCGIS software (ESRI, v. 9.3.1). A signature records the image properties of a defined, discrete area, which can then be used to characterize similar areas. Independent signatures were calculated for each NPA, and in some cases different areas within an NPA, thereby allowing us to take into account differences in resolution and arboreal expression both within and between NPAs. These signatures were subsequently used to classify the $625 \mathrm{~km}^{2}$ area from each NPA using a maximum likelihood analysis. Classifications were then re-examined visually to ensure maximum accuracy. Clouds and aquatic bodies were removed from the area, and the relative percentages of the four densities of tree cover calculated on the basis of this classification. We tested the accuracy of our vegetation classification by comparing our vegetation categories with the principal components of climatic data for the geographical locations of each park (climatic indices were extracted for each national park from the WORLDCLIM dataset; Hijmans et al., 2004). The results of these analyses are shown in Appendix S1.

Each NPA was also assigned to a specific biome category (see Appendix S1) by the interaction of locality data point with the polygon extracted from WWF world ecoregion map (Olson et al., 2001). Olson et al. (2001, p. 933) defined 14 biomes (or ecoregions) as 'large units of land containing a distinct assemblage of natural communities and species, with boundaries that approximate the original extent of natural communities prior to major land-use change'. This categorization will be used to explore the concept of community convergence (see the next section).

\section{Multivariate analyses}

Each NPA is represented by a multivariate dataset for both fauna and arboreal heterogeneity. For mammal communities guild representation is quantified as the percentage of species in a given guild in a NPA; relative percentages of tree cover categories were used for arboreal heterogeneity. Principal components analyses (PCA; Hair et al., 2005) were run for the faunal and vegetation variables separately in order to first explore the datasets. This methodology reduces the dimensionality of the data and allows for examination of potential clustering without any a priori hypothesis.

Synecological analyses require a correlation between environmental and faunal variables so that palaeohabitats can be reconstructed with a degree of accuracy. Consequently, a canonical correlations analysis (COR) was run to check the potential correlation between the full faunal multivariate dataset and the vegetation multivariate dataset. In this type of canonical ordination, faunal canonical scores are the parameters estimated by a multiple regression of the NPA scores on the faunal variables (Jongman et al., 1995). A nested analysis of the separate continents was also run. However, as COR requires that the number of cases (i.e. NPAs in this analysis) be greater than the number of combined variables from both the vegetation and faunal sets of variables, we ran a redundancy analysis (RDA) to analyse the nested values from the individual continents. These two methods use very similar ordination algorithms; however, while the faunal-vegetational correlation is maximized in the COR, it is only produced as a by-product of the RDA algorithm (Jongman et al., 1995). The RDA of the global dataset was compared with the COR results, allowing for comparisons between nested and global results.

Communities that converge in structure will share the same position in multidimensional ecological space (Rodríguez et al., 2006). In order to explore community convergence we conducted two separate principal components analyses on the faunal ecovariables from our NPAs. In order to explore the influence of historical factors, we ran the first PCA using the residuals calculated from a regression between the faunal ecovariables and $\%$ Heavy (the proportion of the NPA covered by continuous 
a)

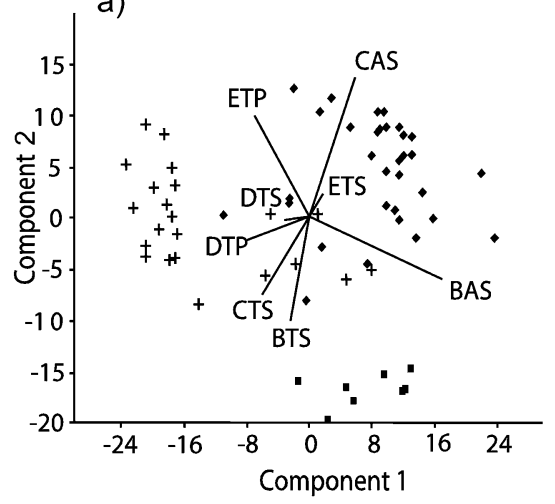

c)

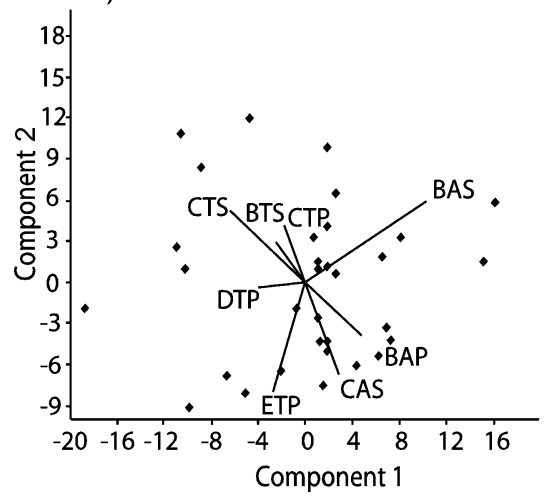

b)

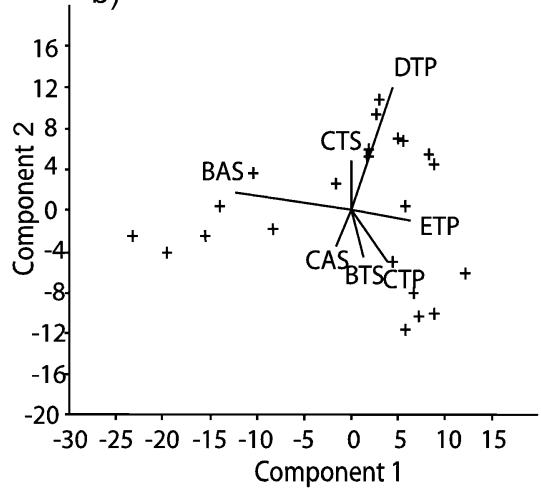

d)

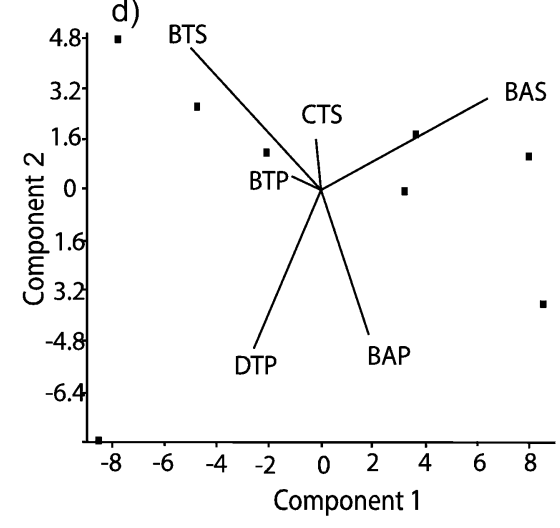

Figure 2 Principal components analysis and biplot of the natural protected areas (NPAs) on the basis of faunal ecological categories: (a) all natural protected areas; (b) African protected areas; (c) Asian protected areas; (d) South and Central American protected areas. Crosses represent African NPAs, diamonds Asian NPAs and squares American NPAs. Abbreviations: B, small; C, medium; D, large; E, very large; A, arboreal; T, terrestrial; $\mathrm{P}$, primary consumer; $\mathrm{S}$, secondary consumer. Only the most significant ecological guild axes are shown in this biplot. canopy cover). We also ran a second PCA using the residuals calculated from a regression between the faunal ecovariables and longitude to explore the influence of environmental factors. While examining just longitude does not give a precise position in space, it does give an approximation of the differences in location between the continents; especially as all our NPAs are found at relatively low latitudes. Because none of the continents we examined straddle the $-180^{\circ} / 180^{\circ}$ longitude line, any potential problems which might result from examining NPAs on either side of this line was avoided. This proxy for geographical position will emphasize inter-continental while minimizing intra-continental differences. This allows us to explore the influence of continent on mammal community structure. Natural protected areas were grouped according to continent and biome for analyses one and two, respectively. If historical factors operate, continents should differ in fauna after removing the effect of vegetation. The same applies for environmental factors that operate if biomes differ in fauna after removing the effect of longitude.

\section{RESULTS}

Linear regression of principal component (PC) 1 from vegetation and fauna against log area for each protected area, with outliers removed (Pasoh Virgin Jungle Reserve and Bukit Kutu Wildlife Reserve), showed no significant correlations (vegetation $P=0.842$; fauna $P=0.145$ ). Faunal PCA results are provided in Fig. 2 and Appendix S2. The first three faunal PC axes explain
$83 \%$ of the variability in global the faunal sample. In particular, PC1, strongly associated with BAS (small, arboreal secondary consumers), separates most African NPAs from the other continents. On the other hand, the second PC is positively loaded by CAS (medium, arboreal secondary consumers) and separates the Americas (negative scores) from the rest (Fig. 2a). BAS is strongly associated with PC1 in both Asia and the American continents (Fig. 2c,d), while Africa PC1 is positively influenced by ETP (very large, terrestrial primary consumers) and CTP (medium, terrestrial primary consumers), with BAS showing the strongest negative association (Fig. 2b). Arboreal heterogeneity PCA results are provided in Table 2 . The first two PC axes explain $95 \%$ of the variability in the vegetation sample. The first PC separates most African NPAs from the other continents. The most significant loading on PC1 is \%Heavy.

The COR and RDA results are summarized in Appendix S2. There is a statistically significant correlation between the global ecospace described by the vegetation and the global ecospace described by the faunal community $\left(r^{2}=0.851\right.$, d.f. $=60,174, P$ $<0.0001)$. An examination of the loadings of each of the vegetation and community variables reveals which ecological group is most closely associated with a particular arboreal heterogeneity (Appendix S2). The strongest vegetation loadings for the significant canonical coefficient (CC1) are percentage of heavy tree cover (canonical loading 0.998 ) and percentage of absent tree cover (canonical loading -0.633). The largest faunal loadings for $\mathrm{CC} 1$, and therefore associated with heavy tree cover, are from small (1-10 kg) arboreal-scansorial secondary consumers 
Table 2 Eigenvalues, loadings and variance explained for the first two principal components (PC) of arboreal heterogeneity variables, calculated for all natural protected areas (NPAs).

\begin{tabular}{lcc}
\hline & PC1 & PC2 \\
\hline Eigenvalue & 1964.39 & 840.248 \\
\% variance explained & 66.394 & 28.399 \\
\%Heavy & 0.8193 & -0.1553 \\
\%Moderate & -0.04017 & 0.1054 \\
\%Light & -0.2809 & 0.719 \\
\%Absent & -0.4982 & -0.6692 \\
\hline
\end{tabular}

\%Heavy refers to the proportion of the NPA covered by continuous canopy cover. \%Moderate, \%Light and \%Absent are categories corresponding to decreases in canopy cover. See Appendix S1 for a schematic representation of the different categories.

(canonical loading 0.908). Species falling into this category include some members of the order Carnivora (e.g. Felidae, Herpestidae, Mustelidae and Viverridae), but also primates and rodents (Muridae and Sciuridae). All but one arboreal category (EAS, > $180 \mathrm{~kg}$, arboreal-scansorial secondary consumers) show positive loadings on CC1. Very large, terrestrial secondary consumers also positively load the first canonical vector. This ecological category includes several ursids (bears) and suids (pigs). Conversely, the strongest negative loading, and hence association with absent or light tree cover, is from large terrestrial primary consumers (canonical loading 0.782 ).

ANOVA performed on loadings of COR and the RDA analyses (both total and nested) showed no significant differences between these values ( $F=0.351$, d.f. $=4,90, P=0.843)$. Consequently, both COR and nested RDA can be equivalently interpreted. The first eigenvalue in each RDA accounts for the majority of variation in the sample size (Africa 0.921, Asia 0.988, America 0.986). The RDA of African protected areas shows equivalent principal loadings as for the COR (Appendix S2). Similar loading regimes exist between the COR and the RDA of Asian NPAs. The only major difference in Asia exists in the loading associated with \%Heavy. In both the global and African datasets this loading correlates most strongly with small arboreal secondary consumers. In Asia it is associated with small arboreal primary consumers and medium arboreal secondary consumers. For America, the loading on \%Heavy is also more strongly associated with small arboreal primary consumers, though the loading on small, arboreal secondary consumers (BAS) is almost equivalent. \%Absent (the proportion of the NPA lacking canopy cover) is the primary loading on the vegetation variables for the Asian NPAs, while for the COR and African NPAs it is second to $\%$ Heavy. Due to the absence of significant open areas in American NPAs, this variable contributes very little to the vegetation loadings in this continent. The second principal loading for vegetation in America is \%Moderate (the proportion of the NPA with moderate canopy cover), and it is associated with small terrestrial secondary consumers.
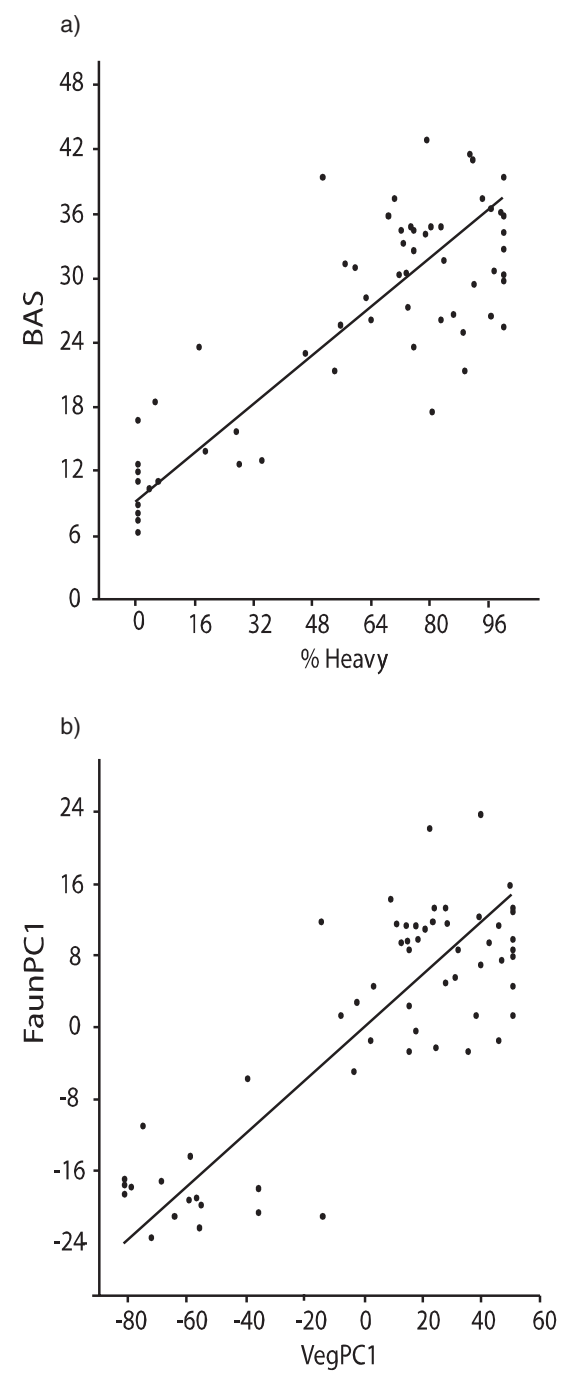

Figure 3 Linear regression between vegetation and fauna. (a) Small, arboreal secondary consumers (BAS) against relative proportion of heavy tree cover (\%Heavy) $(r=0.83779, P<0.001)$. (b) First principal component (PC1) of vegetation and faunal ecospaces $(r=0.84657, P<0.001)$. This figure shows that there is a significant relationship between BAS and the proportion of continuous tree cover in national protected areas (a) and between the faunal and vegetation ecospaces (b).

The canonical $R^{2}$ of the COR $(0.851)$ is very close to the $R^{2}$ value of \%Heavy (0.849), and, to a lesser extent, the $R^{2}$ of BAS (0.71) (see Appendix S2). This means that the global set of variables can be approximated by a comparison between \%Heavy tree cover and BAS. A plot of BAS values against \%Heavy yields a statistically significant, linear relationship (Fig. 3a). Furthermore, the first PC of vegetation has a statistically significant, linear relationship with the first PC of the ecological guilds correlates (Fig. $3 \mathrm{~b}$ ). The second highest $R^{2}$ value is for large, terrestrial primary consumers (DTP). An examination of the nested results (Appendix S2) shows that this variable significantly correlates with \%Absent for all continents except America. 


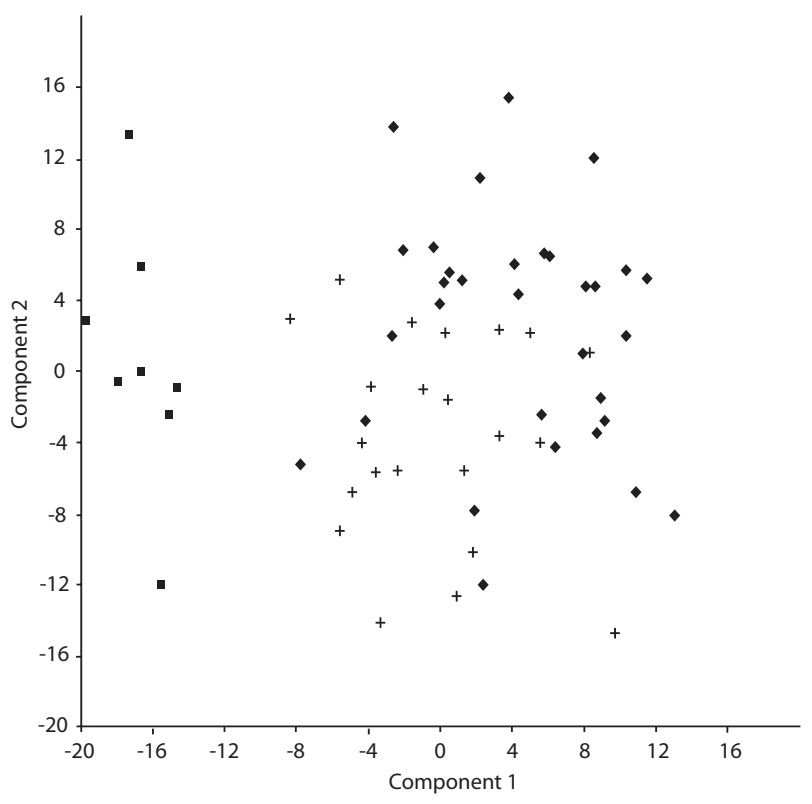

Figure 4 Principal components analysis 1. In this analysis, we corrected for habitat (a proxy for environmental factors), allowing us to test the influence of history on structuring communities. The figure shows the plot of principal component 1 (PC1) against PC2 for the residuals calculated by a regression between the faunal ecological guilds and the proportion of heavy tree cover (\%Heavy) for each natural protected area (NPA). If mammal community structure differs between continents once environment has been accounted for, this would suggest that historical differences between continents are important in structuring communities. Conversely, if there is no separation between continents, this suggests that other factors are involved in structuring communities. Squares represent South and Central America, diamonds Asia and crosses Africa. South and Central American communities are distinctly to the left of the Asian and African communities. This suggests that historical affects have contributed to the structure of South and Central American communities, but not to Asian and African communities.

The first convergence analysis, examining historical differences in community structure, is represented by the PCA of the residuals calculated from a regression between the faunal ecovariables and \%Heavy tree cover (Fig. 4, Table 3). All American NPAs are distinguished from the Asian and African NPAs along the first principal component, which explains $36 \%$ of the variance. Asian and African NPAs show a remarkable convergence in community structure. The second convergence analysis, examining environmental differences in community structure, is represented by the PCA of the residuals calculated from a regression between the faunal ecovariables and longitude (Fig. 5, Table 3). All but one NPA from 'forested' type biomes show the same or very similar structure regardless of continent. They are clearly separated out from most 'open' type NPAs by the first principal component, which explains $63 \%$ of the variance. The only exception is Cape West Coast Biosphere Reserve, which groups with 'open' biomes. However, it is noteworthy that we record zero \%Heavy tree cover for this NPA. There are a number of 'open' biomes which group with the 'forest' biomes. Equally, examining the \%Heavy tree cover for these NPAs shows that all but one have significant proportions of heavy tree coverage (Parque Nacional Canaima 94\%, Río Negro National Park 59\%, Wolong Nature Reserve 56\%; see Appendix S1). The only exception is Parc National de la Comoe, where only $16 \%$ of the park is classified as having heavy tree cover.

\section{DISCUSSION}

There is no doubt that mammal community structure is strongly influenced by tree cover (e.g. Williams \& Marsh, 1998; Perault \& Lomolino, 2000; Williams et al., 2002), and our results provide support that this pattern is consistent across continents. As such, they form a solid baseline for conservation efforts as well as for reconstructing past environments. Having established that community structure and vegetation heterogeneity are significantly correlated means we could examine whether community convergence between geographically disparate communities sharing similar vegetation structure occurs. We found that community convergence was evident in all continents once geographical position had been controlled for. We also found that historical factors are likely to have been similar for Africa and Asia but different for South and Central America. South America has been separated geologically from Africa for between 120 and 90 million years (Krause et al., 2007), although some faunal exchange between the Old and the New World must have occurred after that, probably during the Late Eocene (Gingeritch, 1981). On the other hand, Asia and Africa share a much more recent biogeographical history, with the fossil record showing repeated exchanges of mammals between these two continents over at least the past 5 million years (O'Regan et al., 2010). This suggests that community convergence resulting from environmental factors may operate on a different, and probably shorter, timescale than that of historical factors.

Although community convergence has been assumed by numerous palaeoecologists in reconstructing palaeohabitats (e.g. Kay \& Madden, 1997; Reed, 1997, 1998; Mendoza et al., 2005; Reed \& Russak, 2009), this assumption has recently been questioned. Rodríguez et al. (2006) reported that no direct correspondence existed between biome (as a proxy for environment) and community structure in an analysis based on diversity within ecological guilds in the Holarctic zone. Although they found some convergence, in particular between communities in extreme environments, they noted that historical factors were at least equally as significant as habitat in structuring communities. Interestingly, Kelt et al. (1996) suggested that desert mammalian community structure was strongly contingent on historical factors, although Rodríguez et al. (2006) and Kelt et al. (1996) use different definitions of community convergence. Our study, using the same definition of convergence as Rodríguez et al. (2006) (although only similar and less detailed ecological guilds), shows that while both historical and environmental factors structure communities, the effects of environment may act more quickly on a community than those of historical factors, although both are clearly influential. Although we do 
Table 3 Principal components analysis results for the convergence analyses.

\begin{tabular}{|c|c|c|c|c|c|c|}
\hline & \multicolumn{4}{|c|}{ Analysis 1 (Convergence based on history, Fig. 4) } & \multicolumn{2}{|c|}{$\begin{array}{l}\text { Analysis } 2 \text { (Convergence based } \\
\text { on environment, Fig. 5) }\end{array}$} \\
\hline & $\mathrm{PC} 1$ & PC2 & PC3 & PC4 & $\mathrm{PC} 1$ & PC2 \\
\hline Eigenvalue & 66.853 & 46.999 & 22.092 & 13.714 & 159.446 & 26.253 \\
\hline \%variance & 35.941 & 25.268 & 11.877 & 7.373 & 62.855 & 10.349 \\
\hline ResBAP & -0.068 & 0.110 & -0.072 & 0.696 & -0.154 & 0.237 \\
\hline ResBAS & -0.291 & 0.630 & -0.522 & -0.177 & -0.790 & 0.085 \\
\hline ResBTP & -0.015 & 0.150 & 0.121 & 0.099 & -0.029 & -0.063 \\
\hline ResBTS & -0.448 & 0.031 & 0.407 & 0.222 & 0.009 & -0.448 \\
\hline ResCAP & 0.002 & -0.002 & 0.035 & -0.051 & -0.009 & -0.073 \\
\hline ResCAS & 0.602 & 0.297 & 0.232 & -0.307 & -0.127 & 0.247 \\
\hline ResCTP & -0.056 & -0.020 & 0.487 & -0.071 & 0.073 & -0.326 \\
\hline ResCTS & -0.319 & -0.434 & 0.012 & -0.387 & 0.207 & -0.379 \\
\hline ResDAP & 0.003 & 0.014 & -0.004 & 0.038 & -0.009 & 0.003 \\
\hline ResDAS & 0.010 & -0.019 & 0.051 & -0.140 & -0.023 & 0.010 \\
\hline ResDTP & -0.076 & -0.381 & -0.423 & -0.149 & 0.338 & -0.037 \\
\hline ResDTS & -0.009 & -0.155 & -0.082 & 0.037 & 0.142 & 0.036 \\
\hline ResEAS & 0.092 & -0.010 & 0.018 & -0.081 & 0.035 & 0.026 \\
\hline ResETP & 0.466 & -0.327 & -0.247 & 0.342 & 0.386 & 0.641 \\
\hline ResETS & 0.107 & 0.114 & -0.013 & -0.072 & -0.049 & 0.039 \\
\hline
\end{tabular}

The first analysis explores the influence of history by correcting for habitat (i.e. using the residuals (Res) calculated from a regression between the ecological guilds and \%Heavy, the relative proportion of heavy tree cover). The second analysis explores the influence of environment by correcting for geography (i.e. using the residuals (Res) calculated from a regression between the ecological guilds and longitude). The number of principal components retained was determined by examining the respective scree plots of the different analyses.

Abbreviations: Res, residual; B, small; C, medium; D, large; E, very large; A, arboreal; T, terrestrial; P, primary consumer; S, secondary consumer.

not examine species richness, our study has implications for the debate about whether environment or history is the primary driver of richness (see, for example, Ricklefs, 1987, 2004, 2007; Kerr \& Packer, 1997; Francis \& Currie, 1998, 2003; Kerr et al., 2001; Currie et al., 2004; Qian \& Ricklefs, 2004). Our study suggests that these different factors may be operating on different temporal scales.

However, we need to take into account potential biases that may have an impact on our interpretations. First, our analysis was purposely conducted on a biased sample of NPAs. We targeted ecosystems of high diversity that exclude extreme environments such as deserts or the high Arctic. By excluding less diverse protected areas and only examining proportional representation, we are attempting to minimize the effects of increased diversity between ecosystems or continents, thereby making the NPAs as comparable as possible. In addition, areas of high diversity are most useful for the methods employed herein (Louys et al., 2009). However the criterion that an NPA contains over 32 species of medium- and large-bodied species means we did not sample equivalently across all continents, or even within habitat types in a continent. The diversity threshold used results in a focus upon the tropical belt, an area of higher diversity than other zones. In addition, most of the NPAs represent dense forests, such as rain forests. The only continent which had significant numbers of high-diversity NPAs associated with treeless habitats was Africa. Consequently, the samples chosen have been, to a certain extent, dictated by historical factors such as the
Late Pleistocene extinction of megafauna - likely minimal in Africa (Martin, 1966; Barnosky et al., 2004) and to a lesser extent Asia (Louys et al., 2007; Louys, 2008; Corlett, 2010), but profound in South and Central America (Barnosky et al., 2004; Cione et al., 2009). However, this bias is intentional: it allows us to specifically target the nature of guild structure in faunally diverse ecosystems, a sometimes neglected ecosystem in intercontinental studies (Corlett \& Primack, 2006). Moreover, our study examines areas that were not covered by ice-sheets during glacial periods, allowing us to avoid the problems of comparing forests and grasslands from very different climatic regimes (e.g. tropical versus temperate) as well as those that experienced extreme ecological changes as a result of glaciations.

Second, we acknowledge that our analysis of the vegetation of an NPA may not be truly representative of the vegetation regime of the entire NPA. However, in many cases the mammal community recorded for an NPA corresponds only to the main, and sometimes even just a part, of the vegetation regime of the NPA (Louys et al., 2009). Moreover, although many of the NPAs we examined have an area larger than $650 \mathrm{~km}^{2}$ (the area we sampled), others did not. Pasoh Virgin Jungle Reserve, for example, which records a total of 62 species of mammals, is only $1.08 \mathrm{~km}^{2}$. Using the strict boundaries of this reserve would not capture an area capable of accommodating all the species listed. We further note that although the mammal communities we examined were recorded in the NPAs, most of these species will not be strictly restricted to the area delineated by its boundaries. 




Figure 5 Principal components analysis 2. In this analysis, we corrected community structure for geographical position (a proxy for history), which allowed us to explore the influence of environmental factors. The figure shows the plot of principal component 1 (PC1) against PC2 for the residuals calculated by a regression between the faunal ecological guilds and longitude for each natural protected area (NPA). If mammal community structure separates between broad environmental categories (defined after Olson et al., 2001), this suggests that environmental differences are important in structuring communities. On the other hand, if there is no separation between habitats, this suggests that other factors are involved. Diamonds represent tropical and subtropical moist broadleaf forests; crosses represent tropical and subtropical dry broadleaf forests; oval represents Mediterranean forests, woodlands, and scrub; triangles represent tropical and subtropical grasslands, savannas, and shrublands; squares represent flooded grassland and savannas; asterisk represents montane grasslands and shrublands; rectangles represent temperate broadleaf and mixed forests; and crosses represent deserts and xeric shrublands. This figure shows that almost all parks and reserves show similar structure depending on habitat and regardless of continent, suggesting that environmental effects are influencing community structure.

Although park boundaries sometimes coincide with natural ecotones, they are not ecological entities in their own right. Rather they have been delineated for political or historical reasons. By sampling equivalently across all NPAs, we ensure maximum comparability between NPAs from different countries subject to different policies and regulations. Furthermore, by targeting the NPA's geographical coordinates, we maximize the likelihood that we sample within the NPA's zone A, the central area of a nature reserve which is the most protected. We do note that in certain heavily forested NPAs we recorded some treeless areas. These probably represent newly opened areas resulting from illegal logging or urbanization. Such areas were not excluded as the areas captured in our study will nevertheless potentially be at the disposal of the mammalian community under question, regardless of whether the mammals in question use this space transitively or intensely.

Despite the influence of historical factors in the structuring of communities we can demonstrate a significant correlation between tree cover heterogeneity and ecological guilds. The strongest correlation between the two sets of variables was between the percentage of heavy tree cover and small, arboreal secondary consumers which occurs in the global and in the African dataset. On the other hand, DTP (large primary terrestrial consumers) exhibits the most dominant correlation within the datasets encompassing all continents except America. Clearly, the guild of secondary arboreal consumers necessitates tree cover and its relationship with vegetation heterogeneity is not unexpected. This group of animals (mostly carnivores belonging to Herpestidae and Viverridae but also some felids and primates) seems to be informative predominantly for the African continent. Conversely, medium-sized ungulates show a high degree of association with vegetation cover in all cases except America. Ungulates are commonly used to reconstruct past environments, and previous studies have demonstrated that several parameters of ungulate community (e.g. mean hypsodonty; Fortelius et al., 2006) are associated with the physical environment. Here, we demonstrate that the simple proportion of large-bodied, terrestrial primary consumers is directly associated with degree of tree coverage across continents.

These relationships are of particular relevance to palaeoecological reconstructions. The relationship which exists between the vegetation and faunal ecospaces can be used to reconstruct the vegetational ecospace on the basis of fossil communities, and we are currently formulating and testing this potential (J.L. et al., unpublished data). These relationships also allow us to predict the effects of deforestation or fragmentation on the entire mammal community, a calculation with obvious applications to conservation.

\section{CONCLUDING REMARKS}

Our results show a significant correlation between vegetation heterogeneity and mammal community structure. The most consistent guild associated with arboreal heterogeneity across continents was that of large-bodied, terrestrial primary consumers. Unsurprisingly, a greater proportion of these are found in areas with fewer trees. Less expected was the global association of small arboreal-scansorial secondary consumers with heavy tree cover. The correlations calculated herein have important implications for conservation efforts. Using the relationships we describe, the likely effects of deforestation on mammal community structure can be predicted. Globally, small, arboreal and semi-arboreal secondary consumers are the most strongly associated with proportion of heavy tree cover in NPAs. The loss of large tracts of dense forests will not only affect strictly arboreal folivorous or frugivorous species, but they will also affect, and perhaps more significantly, small, omnivorous and carnivorous arboreal and scansorial mammals. These results confirm the overall scenario for most mammals threatened with extinction (e.g. most of the arboreal primates are likely to be affected by 
habitat loss; Schipper et al., 2008). In support of our findings, Cardillo et al. (2004) point out that small mammalian carnivores such as African viverrids are quite sensitive to human expansion and habitat loss.

The global association between vegetation and community structure can also allow palaeoecologists to reconstruct likely vegetation heterogeneity on the basis of fossil communities. The use of the relationships detailed herein will allow an examination of changes in community structure through time. The further back in time one goes, and the less that is known about individual extinct species, the more important communitybased analyses become. Our results demonstrate that while both historical and environmental factors play a role in structuring mammal communities in diverse ecosystems, environmental factors may act on a more rapid timescale than historical. In particular, South and Central American sites show differences in community structure compared with African and Asian ones, which do not. All three continents show similar structure according to habitat. This confirms that synecological reconstructions of fossil sites will provide an accurate reflection of the environments present when they were deposited. However understanding historical and environmental influences, both spatially and temporally, is integral to synecological habitat reconstructions. Because of the inter-twined nature of both historical and environmental effects on structuring communities, we suggest that a global approach to synecological habitat reconstructions may capture more of the variability possible in fossil communities than restricting such analyses to the region where such fossils are found. Because historical factors appear to operate on large temporal scales, such a holistic approach will be integral to reconstructions made for fossil sites in deep time. Understanding how changes in vegetation affect community structure, both in the past and now, is integral in helping us combat the global loss of species through habitat alteration and destruction.

\section{ACKNOWLEDGEMENTS}

We thank two anonymous referees and D. Currie and T. Blackburn for useful suggestions which greatly improved this paper. D. Wilkinson provided valuable comments on an earlier draft. We thank The Leverhulme Trust for generous support of this research $(\mathrm{F} / 00,754 / \mathrm{C})$.

\section{REFERENCES}

Andrews, P.J. (1989) Palaeoecology of Laetoli. Journal of Human Evolution, 18, 173-181.

Andrews, P.J. (1996) Paleoecology and hominid palaeoenvironments. Biological Reviews, 71, 257-300.

Andrews, P.J., Lord, J.M. \& Nesbit-Evans, E.M. (1979) Patterns of ecological diversity in fossil and modern mammalian faunas. Biological Journal of the Linnean Society, 11, 177-205.

August, P.V. (1983) The role of habitat complexity and heterogeneity in structuring tropical mammal communities. Ecology, 64, 1495-1507.
Bakker, V.J. \& Kelt, D.A. (2000) Scale-dependent patterns in body size distributions in Neotropical mammals. Ecology, 81, 3530-3547.

Barnosky, A., Koch, P., Feranec, R., Wing, S. \& Shabel, A. (2004) Assessing the causes of Late Pleistocene extinctions on the continents. Science, 306, 70-75.

Blondel, J. (1991) Assessing convergence at the communitywide level. Trends in Ecology and Evolution, 6, 271-272.

Blondel, J. (2003) Guilds or functional groups: does it matter? Oikos, 100, 223-231.

Cardillo, M., Purvis, A., Sechrest, W., Gittleman, J.L., Bielby, J. \& Mace, G.M. (2004) Human population and extinction risk in the world's carnivores. PLoS Biology, 2, 909-914.

Cione, A.L., Tonni, E.P. \& Soibelzon, L. (2009) Did humans cause the Late Pleistocene-Early Holocene mammalian extinctions in South America in a context of shrinking open areas. American megafaunal extinctions at the end of the Pleistocene (ed. by G. Haynes), pp. 125-144. Springer Science, Netherlands.

Corlett, R.T. (2010) Megafaunal extinctions and their consequences in the tropical Indo-Pacific. Altered ecologies: fire, climate and human influence on terrestrial landscapes (ed. by S.G. Haberle, J. Stevenson and M. Prebble), pp. 117-132. Terra Australis 32. ANU E-Press, Canberra.

Corlett, R.T. \& Primack, R.B. (2006) Tropical rainforests and the need for cross-continental comparisons. Trends in Ecology and Evolution, 21, 104-110.

Cristoffer, C. \& Peres, C.A. (2003) Elephants versus butterflies: the ecological role of large herbivores in the evolutionary history of two tropical worlds. Journal of Biogeography, 30, 1357-1380.

Currie, D.J., Mittelbach, G.G., Cornell, H.V., Field, R., Guégan, J.-F., Hawkins, B.A., Kaufman, D.M., Kerr, J.T., Oberdorff, T., O'Brien, E. \& Turner, J.R.G. (2004) Predictions and tests of climate-based hypotheses of broad-scale variation in taxonomic richness. Ecology Letters, 7, 1121-1134.

Danell, K., Lundberg, P. \& Nimelä, P. (1996) Species richness in mammalian herbivores: patterns in the boreal zone. Ecography, 19, 404-409.

Fleming, T.H. (1973) Numbers of mammal species in North and Central American forest communities. Ecology, 54, 555-563.

Fleming, T.H., Breitwisch, R. \& Whitesides, G.H. (1987) Patterns of tropical vertebrate frugivore diversity. Annual Review of Ecology and Systematics, 18, 91-109.

Fortelius, M., Eronen, J.T., Liu, L., Pushkina, D., Tesakov, A., Vislobokova, I.A. \& Zhang, Z. (2006) Late Miocene and Pliocene large land mammals and climatic changes in Eurasia. Palaeogeography, Palaeoclimatology, Palaeoecology, 238, 219 227.

Francis, A.P. \& Currie, D.J. (1998) Global patterns of tree species richness in moist forests: another look. Oikos, 81, 598-602.

Francis, A.P. \& Currie, D.J. (2003) A globally consistent richness-climate relationship for angiosperms. The American Naturalist, 161, 523-536.

Fuentes, E.R. (1976) Ecological convergence of lizard communities in Chile and California. Ecology, 57, 3-17. 
Gingeritch, P.D. (1981) Eocene Adapidae, paleobiogeography, and the origin of South American Platyrrhini. Evolutionary biology of the New World monkeys and continental drift (ed. by R. L. Ciochon and B. Chiarelli), pp. 123-138. Plenum Publishing Corporation, New York.

Hair, J.F., Black, B., Babin, B., Anderson, R.E. \& Tatham, R.L. (2005) Multivariate data analysis, 6th edn. Prentice Hall, Upper Saddle River, NJ.

Hijmans, R.J., Guarino, L., Bussink, C., Mathur, P., Cruz, M., Barrentes, I. \& Rojas, E. (2004) DIVA-GIS. Vsn. 5.0. A geographic information system for the analysis of species distribution data. Available at: http://www.diva-gis.org (accessed 29 September 2010).

Jongman, R.H.G., ter Braak, C.J.F. \& van Tongeren, O.F.R. (1995) Data analysis in community and landscape ecology. Cambridge University Press, Cambridge.

Kay, R.F. \& Madden, R.H. (1997) Mammals and rainfall: paleoecology of the middle Miocene at La Venta (Colombia, South America). Journal of Human Evolution, 32, 161199.

Kelt, D.A., Brown, J.H., Heske, E.J., Marquet, P.A., Morton, S.R., Reid, J.R.W., Rogovin, K.A. \& Shenbrot, G. (1996) Community structure of desert small mammals: comparisons across four continents. Ecology, 77, 746-761.

Kerr, J.T. \& Packer, L. (1997) Habitat heterogeneity as a determinant of mammal species richness in high energy regions. Nature, 385, 252-254.

Kerr, J.T., Southwood, T.R.E. \& Cihlar, J. (2001) Remotely sensed habitat diversity predicts butterfly species richness and community similarity in Canada. Proceedings of the National Academy of Sciences USA, 98, 11365-11370.

Kovarovic, K., Andrews, P. \& Aiello, L. (2002) The palaeoecology of the Upper Ndolanya Beds at Laetoli, Tanzania. Journal of Human Evolution, 43, 395-418.

Krause, D.W., Sampson, S.D., Carrano, M.T. \& O’Connor, P.M. (2007) Overview of the history of discovery, taxonomy, phylogeny, and biogeography of Majungasaurus crenatissimus (Theropoda: Abelisauridae) from the Late Cretaceous of Madagascar. Journal of Vertebrate Paleontology, 27(Suppl. 2), $1-20$.

Louys, J. (2008) Quaternary extinctions in Southeast Asia. Mass extinction (ed. by A.M.T. Elewa), pp. 159-189. SpringerVerlag, Heidelberg.

Louys, J. \& Meijaard, E. (2010) Palaeoecology of Southeast Asian megafauna-bearing sites from the Pleistocene and a review of environmental changes in the region. Journal of Biogeography, 37, 1432-1449.

Louys, J., Curnoe, D. \& Tong, H. (2007) Characteristics of Pleistocene megafauna extinctions in Southeast Asia. Palaeogeography, Palaeoclimatology, Palaeoecology, 243, 152-173.

Louys, J., Travouillon, K.J., Bassarova, M. \& Tong, H. (2009) The use of protected natural areas in palaeoecological analyses: assumptions, limitations and application. Journal of Archaeological Science, 36, 2274-2288.

Martin, P.S. (1966) Africa and Pleistocene overkill. Nature, 212, 339-342.
Mendoza, M., Goodwin, B. \& Criado, C. (2004) Emergence of community structure in terrestrial mammal-dominated ecosystems. Journal of Theoretical Biology, 230, 203-214.

Mendoza, M., Janis, C.M. \& Palmqvist, P. (2005) Ecological patterns in the trophic-size structure of large mammal communities: a 'taxon-free' characterization. Evolutionary Ecology Research, 7, 505-530.

Nesbit-Evans, E.M., van Couvering, J.A.H. \& Andrews, P. (1981) Palaeoecology of Miocene sites in Western Kenya. Journal of Human Evolution, 10, 35-48.

Nowak, R.M. (1999) Walker's mammals of the world. The Johns Hopkins University Press, London.

O’Regan, H.J., Turner, A., Bishop, L.C., Elton, S. \& Lamb, A. (2010) Hominins without fellow travellers? First appearances and inferred dispersals of Afro-Eurasian large-mammals in the Plio-Pleistocene. Quaternary Science Reviews, doi:10.1016/ j.quascirev.2009.11.028.

Olff, H., Ritchie, M.E. \& Prins, H.H.T. (2002) Global environmental controls of diversity in large herbivores. Nature, 415, 901-904.

Olson, D.M., Dinerstein, E., Wikramanayake, E.D., Burgess, N.D., Powell, G.V.N., Underwood, E.C., D’Amico, J.A., Itoua, I., Strand, H.E., Morrison, J.C., Loucks, C.J., Allnutt, T.F., Ricketts, T.H., Kura, Y., Lamoreux, J.F., Wettengel, W.W., Hedao, P. \& Kassem, K.R. (2001) Terrestrial ecoregions of the world: a new map of life on earth. BioScience, 51, 933-938.

Perault, D.R. \& Lomolino, M.V. (2000) Corridors and mammal community structure across a fragmented, old-growth forest landscape. Ecological Monographs, 70, 401-422.

Qian, H. \& Ricklefs, R.E. (2004) Taxon richness and climate in angiosperms: is there a globally consistent relationship that precludes region effects? The American Naturalist, 163, 773779.

Reed, K.E. (1997) Early hominid evolution and ecological change through the African Plio-Pleistocene. Journal of Human Evolution, 32, 289-322.

Reed, K.E. (1998) Using large mammal communities to examine ecological and taxonomic structure and predict vegetation in extant and extinct assemblages. Paleobiology, 24, 384408.

Reed, K.E. \& Russak, S.M. (2009) Tracking ecological change in relation to the emergence of Homo near the Plio-Pleistocene boundary. The first humans: origin and early evolution of the genus Homo (ed. by F.E. Grine, J.G. Fleagle and R.E. Leakey), pp. 159-171. Springer Science, Dordrecht.

Ricklefs, R.E. (1987) Community diversity - relative roles of local and regional processes. Science, 235, 167-171.

Ricklefs, R.E. (2004) A comprehensive framework for global patterns in biodiversity. Ecology Letters, 7, 1-15.

Ricklefs, R.E. (2007) History and diversity: explorations at the intersection of ecology and evolution. The American Naturalist, 170, S56-S70.

Rodríguez, J. (2004) Stability in Pleistocene Mediterranean mammalian communities. Palaeogeography, Palaeoclimatology, Palaeoecology, 207, 1-22. 
Rodríguez, J. (2006) Structural continuity and multiple alternative stable states in Middle Pleistocene European mammalian communities. Palaeogeography, Palaeoclimatology, Palaeoecology, 239, 355-373.

Rodríguez, J., Hortal, J. \& Nieto, M. (2006) An evaluation of the influence of environment and biogeography on community structure: the case of Holarctic mammals. Journal of Biogeography, 33, 291-303.

Root, R.B. (1967) The niche exploitation pattern of the bluegray gnatcatcher. Ecological Monograph, 37, 317-350.

Samuels, C.L. \& Drake, J.A. (1997) Divergent perspectives on community convergence. Trends in Ecology and Evolution, 12, 427-432.

Schipper, J., Chanson, J., Chiozza, F. et al. (2008) The status of the world's land and marine mammals: diversity, threat, and knowledge. Science, 322, 225-230.

de Vivo, M. \& Carmignotto, A.P. (2004) Holocene vegetation change and the mammal faunas of South America and Africa. Journal of Biogeography, 31, 943-957.

Vrba, E.S. (1980) The significance of bovid remains as indicators of environment and predation patterns. Fossils in the making (ed. by A.K. Behrensmeyer and A. Hill), pp. 247-271. University of Chicago Press, Chicago, IL.

Williams, S.E. \& Marsh, H. (1998) Changes in small mammal assemblage structure across a rain forest/open forest ecotone. Journal of Tropical Ecology, 14, 187-198.

Williams, S.E., Marsh, H. \& Winter, J. (2002) Spatial scale, species diversity, and habitat structure: small mammals in Australian tropical rain forest. Ecology, 83, 1317-1329.

Wilson, J.B. (1989) A null model of guild proportionality, applied to stratification of a New Zealand temperate rain forest. Oecologia, 80, 263-267.

Wilson, J.B. \& Whittaker, R.J. (1995) Assembly rules demonstrated in a saltmarsh community. Journal of Ecology, 83, 801807.

Wilson, J.B., Allen, R.B. \& Lee, W.G. (1995) An assembly rule in the ground and herbaceous strata of a New Zealand rain forest. Functional Ecology, 9, 61-64.

\section{SUPPORTING INFORMATION}

Additional Supporting Information may be found in the online version of this article:

Appendix S1 Ecological guilds for each natural protected area used in the analyses.

Appendix S2 Summary statistics for principal components, canonical correlations and redundancy analyses (PCA, COR and RDA).

As a service to our authors and readers, this journal provides supporting information supplied by the authors. Such materials are peer-reviewed and may be reorganized for online delivery, but are not copy-edited or typeset. Technical support issues arising from supporting information (other than missing files) should be addressed to the authors.

\section{BIOSKETCHES}

Julien Louys is a post-doctoral research assistant at the Research Centre for Evolutionary Anthropology and Palaeoecology at Liverpool John Moores University. His current research interests include developing new approaches in palaeocommunity and vertebrate palaeoecology analyses, as well as palaeoenvironmental reconstructions and the biogeographical history of the African and Asian Plio-Pleistocene.

Author contributions: J.L conceived the study and collected the primary data, J.L and C.M conducted statistical analyses, J.L., C.M., S.E., P.D. and L.C.B. interpreted the results and wrote the paper.

Editor: Tim Blackburn 